\title{
56. SOBRE LA DEMOGRAFÍA, BIOLOGÍA Y CONSERVACIÓN DE CYTISUS MALACITANUS BOISS. SUBSP. MOLEROI (FERN. CASAS) A. LORA, HERN. BERM. Y J. PRADOS (FABACEAE)
}

\author{
Ángel LORA GONZÁLEZ, J. Esteban HERNÁNDEZ BERMEJO y Josefa PRADOS LIGERO
}

Demography, biology and conservation of Cytisus malacitanus subsp. moleroi (Fern. Casas) A. Lora, Hern. Berm. \& J. Prados (Fabaceae).

Palabras clave. Cytisus, demografía, biología, conservación, Málaga, España.

Key words. Cytisus, demography, biology, coservation, Malaga, Spain.

Cytisus malacitanus subsp. moleroi es un taxón endémico del NO de la provincia de Málaga que tras ser descrito en 1980, no volvió a ser hallado hasta 1991. El estudio detallado de los individuos procedentes tanto de las dos localidades primitivamente mencionadas en la Sierra de Cañete (Cañete la Real) así como de las posteriormente citadas en la Sierra de Peñarrubia (Campillos), en la Sierra de la Camorra (Teba) y en Sierra Llana (Valle de Abdalajís), pusieron de manifiesto las semejanzas con las plantas procedentes de las poblaciones de $C$. malacitanus y permitieron proponer la combinación que utilizamos en el presente trabajo (Lora et al., 1998).

Junto con el trabajo de campo, las semillas colectadas para el Banco de Germoplasma Vegetal del Jardín Botánico de Córdoba, hicieron posible iniciar estudios sobre algunos aspectos de la biología y el estado de conservación de un taxón considerado hasta el momento como en peligro inminente de extinción (Hernández Bermejo et al., 1994).

Demografía. Las dos poblaciones publicadas por Fernández Casas (1980 y 1982) estaban constituidas por no más de 250 individuos (unos 200 en una de ellas y menos de 50 en la otra). El trabajo de campo ha permitido localizar tres núcleos demográficos: uno con centro en Sierra Llana (vertiente E-NE de la Sierra del Valle de Abdalajís), otro en la Sierra de Cañete y el tercero en el eje Sierra de la Camorra-Sierra de Peñarrubia, estando estos dos últimos unidos por la cita de un individuo aislado en el Cortijo del Tajo, relicto evidente de una población continua afectada por el cultivo de las zonas planas y menos pedregosas de los respectivos municipios; entre estas dos poblaciones y Sierra Llana se interpone el Embalse del Guadalhorce-Guadalteba, que también ha condicionado la falta de continuidad actual entre todas las localidades detectadas. Se ha efectuado una evaluación demográfica en cada una de las mencionadas localidades, estimándose la población total del taxón en unos 15.000-20.000 individuos (tab. 1).

Fenología. En la tabla 2 se resumen las principales fenofases observadas en campo. Excepto para $C$. malacitanus subsp. malacitanus, con el que coincide sólo parcialmente, para ningún otro taxón de la sect. Sarothamnus se ha descrito una floración plenamente invernal que se produce en un lapso de tiempo de unos tres meses y medio y de forma muy abundante. Resulta también llamativa la larga duración del periodo de fruto

Trabajo elaborado parcialmente con cargo al VII Acuerdo Específico de la A.M.A. de la Junta de Andalucía y el Jardín Botánico de Córdoba sobre Planes de Recuperación de especies amenazadas. 


\begin{tabular}{ccccc}
\hline Localidad & Cuadrícula & & & \\
UTM (30S) & Altitud $(\mathrm{m})$ & Orientación & $\mathrm{N}^{\text {o }}$ de plantas \\
\hline $1^{*}$ & UF1994 & 800 & SO & $2 \times 10^{2}$ \\
2 & UF2091 & 680 & $\mathrm{~S}$ & $2 \times 10^{2}$ \\
3 & UF2090 & 720 & $\mathrm{~S}-\mathrm{SE}$ & $3 \times 10^{2}$ \\
4 & UF2293 & 660 & $\mathrm{~N}$ & $3 \times 10^{3}$ \\
$5^{*}$ & UF1995 & 600 & $\mathrm{O}$ & $4 \times 10^{1}$ \\
6 & UF3694 & $440-700$ & SO & $4 \times 10^{3}$ \\
7 & UF3493 & $400-700$ & $\mathrm{~S}$ & $4 \times 10^{3}$ \\
8 & UF3595 & $560-700$ & $\mathrm{~N}-\mathrm{NE}$ & $4 \times 10^{2}$ \\
9 & UF3294 & 400 & $\mathrm{SE}$ & $5 \times 10^{2}$ \\
10 & UF3094 & $520-700$ & $\mathrm{~S}$ & $5 \times 10^{2}$ \\
11 & UF2995 & $520-700$ & $\mathrm{NE}$ & $1 \times 10^{3}$ \\
12 & UF2895 & 440 & Perfil plano & $2 \times 10^{1}$ \\
13 & UF3096 & $440-700$ & $\mathrm{~N}$ & $1 \times 10^{2}$ \\
14 & UF2693 & 600 & $\mathrm{NE}$ & $1 \times 10^{0}$ \\
15 & UF4191 & 600 & $\mathrm{NE}$ & $5 \times 10^{2}$ \\
16 & UF4391 & $600-800$ & $\mathrm{~N}-\mathrm{NE}$ & $1 \times 10^{3}$ \\
\hline
\end{tabular}

Área de ocupación estimada: $210 \mathrm{Km}^{2}$

Tabla 1. Distribución y tamaño de las poblaciones de Cytisus malacitanus subsp. moleroi. $\left({ }^{*}\right)$ Localidades publicadas anteriormente. Distribution area and size of the populations of Cytisus malacitanus subsp. moleroi $(*)$ Localities already published.

verde con relación a la rápida maduración de las legumbres que, eso sí, ocurre dentro de los límites temporales citados para el resto de los taxones de la sección. La dispersión es bastante rápida y, una vez realizada, la planta prácticamente desaparece del paisaje pues su parte más visible queda reducida a tallos defoliados de aspecto seco y color marrón que se confunden con el suelo o con el matorral circundante.

Este comportamiento fenológico de floración invernal alejado temporalmente del pico de floración primaveral y de mayor duración, junto con la tendencia constatada de que la planta se presenta en núcleos densos, contrasta con la estrategia de $C$. malacitanus subsp. malacitanus, que tiene una floración más coincidente con el mencionado pico, un periodo de floración más breve (Hidalgo y Cabezudo, 1994) y un modelo más disperso de ocupación del territorio. Esto puede ser interpretado de dos maneras distintas: a) Puede constituir el resultado de fenómenos competitivos por la polinización dentro de la comunidad ya que segregarse fenológicamente de la época más propicia para los polinizadores requiere una mayor recompensa para los mismos, ofreciendo mayor cantidad de flores por unidad de superficie y durante más tiempo. Comportamientos similares han sido ya descritos en Andalucía Occidental para otros taxones como Rosmarinus officinalis, Daphne gnidium o Thymbra capitata (Arroyo, 1986) así como en otras regiones de clima mediterráneo (Kummerow, 1983).

b) En la misma línea de optimizar los mecanismos de polinización, puede entenderse como una adaptación del taxón, su coincidencia con la sinusia de la antesis invernal de las comunidades del matorral serial en las que se encuentra, tanto de Genisto umbellataeCytisetum fontanesii Rivas Goday y Rivas Martínez, 1968 (para el subsector Antequerano 


\begin{tabular}{|l|c|c|c|c|c|c|c|c|c|c|c|c|}
\cline { 2 - 9 } \multicolumn{1}{c|}{} & $\mathrm{E}$ & $\mathrm{F}$ & $\mathrm{M}$ & $\mathrm{A}$ & $\mathrm{M}$ & $\mathrm{J}$ & $\mathrm{Jl}$ & $\mathrm{A}$ & $\mathrm{S}$ & $\mathrm{O}$ & $\mathrm{N}$ & $\mathrm{D}$ \\
\hline $\begin{array}{l}\text { Inicio floración } \\
\text { Floración plena } \\
\text { Fruto verde } \\
\text { Fruto maduro } \\
\text { Inicio dispersión } \\
\text { Dispersión plena }\end{array}$ & & & & & & & & & & & & \\
\hline
\end{tabular}

Tabla 2. Fenología de Cytisus malacitanus subsp. moleroi. Phenology of Cytisus malacitanus subsp. moleroi.

del sector Hispalense en las Sierras de Cañete, de la Camorra y de Peñarrubia) como de Teucrio lusitanici-Coridothymetum capitati Rivas Goday y Rivas Martínez, 1968 (para el subsector Torcalense del sector Rondeño en Sierra Llana), pues el taxón obtiene un claro beneficio al ser la única papilionácea de flor amarilla que, en pleno invierno, comparte polinizadores con las demás especies del citado matorral dominante.

Biología. El modelo mencionado de distribución espacial del taxón y la presencia de Apoideos visitando sus flores, nos hizo pensar en un alto grado de polinización por alogamia. No obstante, a fin de estimar la capacidad de reproducción por autogamia en polinización controlada, se embolsaron inflorescencias enteras y flores aisladas con bolsas de nylon de $30 \mu \mathrm{m}$ de luz de malla, en 30 individuos de dos poblaciones diferentes (Sierra de Peñarrubia y Sierra Llana). En ningún caso se produjo desarrollo de frutos.

Según los estudios de Crudent (1977), la proporción entre polen y primordios seminales por flor $(\mathrm{P} / \mathrm{O})$ es un buen indicador del sistema de reproducción que opera en la planta. Del material colectado en campo se determinó el número de primordios seminales (obteniéndose una media de 4,5 con oscilaciones entre 2 y 7 ) y el número de granos de polen. Para esto último se utilizó material de herbario, preparándose suspensiones de 5 $\mathrm{ml}$ de agua jabonosa con lactofenol y anilinaazul como colorante con las 10 anteras de una flor maceradas y homogeneizadas mediante agitador mecánico. Las mediciones se realizaron sobre 15 botones florales pertenecientes a 6 localidades diferentes, extrayéndose 10 muestras por cada tubo de 1 $\mu 1$. La cifra obtenida es de 66.900 (con mínimo de 39.500 y máximo de 108.500). La relación final P/O es 14.867, relación que se corresponde según Crudent (op. cit.) con la existencia de xenogamia, coincidiendo con su comportamiento reproductor de planta alógama deducido de los ensayos de polinización controlada.

$\mathrm{Su}$ tasa de fertilidad, fue evaluada mediante la relación primordios/legumbre y semillas/legumbre. Los datos medios obtenidos sobre 150 muestras pertenecientes a 5 poblaciones diferentes son de un 53,3\% de éxito en la fecundación y desarrollo de los primordios seminales, lo que supone una tasa de fertilidad alta.

Por último, la capacidad de germinación se determinó construyendo las correspondientes curvas para distintos ensayos controlados. Sin hacer ningún tipo de selección, el taxón arroja un porcentaje de germinación alto $(33,3 \%)$ con 


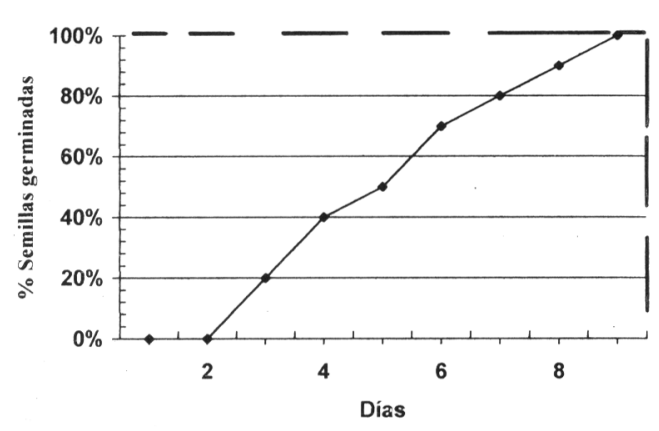

Figura 1. Curva de germinación de Cytisus malacitanus subsp. moleroi. Germination curve of Cytisus malacitanus subsp. moleroi.

semillas dispuestas en placas Petri a temperatura constante $\left(16^{\circ} \mathrm{C}\right)$ con un fotopoeriodo de $16 \mathrm{~h} / 8 \mathrm{~h}$ (luz/oscuridad). Las condiciones óptimas se establecieron con esas mismas variables pero tras ser sometidas a escarificado mecánico, llegándose a obtener porcentajes del $100 \%$ a los 9 días de iniciado el ensayo (Fig. 1).

Conservación. Tal y como ya hemos citado, conforme a las categorías propuestas para el Libro Rojo de Datos de la U.I.C.N. (1981), Cytisus malacitanus subsp. moleroi ha sido considerada hasta el momento como una especie En Peligro (E). Teniendo en cuenta fundamentalmente los efectivos reales del taxón y la eficacia mostrada por su sistema de multiplicación sexual, las amenazas para sus poblaciones vienen dadas sobre todo por la actividad agrícola y ganadera de la zona y, sólo en algunos casos, por la extracción de áridos en algunas canteras próximas tanto a Cañete la Real como a Campillos.

La actividad agrícola -habida cuenta de la naturaleza del suelo calizo, pedregoso y bastante degradado- es marginal en gran parte del territorio y por tanto no constituye una amenaza para la conservación del taxón.

La actividad ganadera es sin embargo más agresiva, ya que la planta resulta evidentemente ramoneada por la abundante cabaña caprina presente en todas las localidades en las que se encuentra, sobre todo durante la sequía estival. A falta de análisis fitoquímicos que 10 confirmen, parece posible la existencia de toxinas tanto en la flor como en la legumbre han sido descritos el alcaloide esparteína y el glucósido escoparina para otras especies del género como C. scoparius (Font Quer, 1980. Rivera y Obón, 1991), con mayor concentración en la planta durante la antesis- que podrían impedir el ataque del ganado hasta que se

\begin{tabular}{cccc}
\hline Accesión & Localidad & Fecha & U.T.M. \\
\hline $14235-92$ & Cañete la Real - Ronda & Mayo de 1992 & 30SUF2090 \\
$14236-92$ & Cañete la Real - Teba & Mayo de 1992 & 30SUF2091 \\
$14237-92$ & Sierra Llana (Sierra del & Mayo de 1992 & 30SUF4191 \\
& Valle de Abdalajís) & & \\
$14543-92$ & Sierra de Peñarrubia & Julio de 1992 & 30SUF3694 \\
$15544-92$ & Cañete la Real & Julio de 1992 & 30SUF1994 \\
$14701-93$ & Almargen & Mayo de 1993 & 30SUF1995 \\
$14699-93$ & Jierra Llana (Sierra del & Junio de 1993 & 30SUF4190 \\
$14700-93$ & Valle de Abdalajís) & Junio de 1993 & 30SUF3694 \\
$14702-93$ & Sierra de Peñarrubia & Junio de 1993 & 30SUF3094 \\
$14703-93$ & Teba & Junio de 1993 & 30SUF1994 \\
\hline
\end{tabular}

Tabla 3. Accesiones de Cytisus malacitanus subsp. moleroi incluidas en el B.G.V. del Jardín Botánico de Córdoba. Accesions of Cytisus malacitanus subsp. moleroi. 
produce la rápida dispersión de las semillas. Sólo a partir de ese momento el ramoneo es eficaz, pero la planta ha conseguido ya completar su ciclo.

Siguiendo los criterios U.I.C.N. publicados en 1994, la corología y demografía completadas en nuestro trabajo nos permiten proponer el cambio de categoría de amenaza a VULNERABLE (VU), pues su área de ocupación no llega a superar los $2.000 \mathrm{Km}^{2}$, dándose además la circunstancia añadida de que se encuentra fragmentada debido a la presencia tanto de zonas cultivadas como del Embalse del Guadalhorce-Guadalteba. También puede constatarse, sin embargo, que por el momento los efectivos no se encuentran en una fase de declinación continua y no se han producido fluctuaciones extremas ni en la extensión de presencia, ni en el área de ocupación, ni en el número de localidades o subpoblaciones ni en el número de ejemplares maduros.

Las medidas concretas de conservación que se pueden proponer no suponen sino continuar con las actualmente en curso: conservación ex situ en Banco de Germoplasma (en la tabla 3 se muestran las accesiones incluidas en el B.G.V. del Jardín Botánico de Córdoba) y conservación in situ de la localidad de Sierra Llana que, en uno de sus extremos, penetra dentro de los actuales límites de un Espacio Natural ya protegido: el Paraje Natural del Desfiladero de los Gaitanes.

\section{BIBLIOGRAFÍA}

ARROYO, J. -1988- Atributos florales fenológicos de la floración en matorrales en el Sur de España. Lagascalia 15(1): 43-78.

CRUDENT, R.W. -1977- Pollen-ovule rations: a conservative indicator of breeding systems in flowering plants. Evolution 31: 32-46.

FERNÁNDEZ CASAS, J. -1980-Exsiccata quaedam a nobis distributia III: 163-253

FERNÁNDEZ CASAS, J. -1982- De flora occidentali, 2. Fontqueria 2: 25-42.

FONT QUER, P. -1980- Plantas medicinales. El Dioscórides renovado. $6^{\text {a }}$ edición. Ed. Labor S.A. Barcelona

HERNÁNDEZ BERMEJO, J.E., M.A. CLEMENTE y A.J. PUJADAS -1994- Catálogo general de las especies de recomendada protección en Andalucía (Endémicas, Raras y Amenazadas de Extinción). In: Hernández Bermejo, J.E. y M. Clemente (Eds.), Protección de la Flora en Andalucia, 43-62. Agencia del Medio Ambiente, Consejería de Cultura y Medio Ambiente, Junta de Andalucía, Sevilla.

HIDALGO, M.I. y B. CABEZUDO - 1994- Fenología y volumen de floración del matorral de la Sierra de Mijas. Act. Bot. Malacitana 19: 123-136.

KUMMEROW, J. -1983- Comparative phenology of Mediterranean type plant communities. In F.J. Kruger, D.T. Mitchell \& J.V.M. Jarvis (Eds.), Ecological studies, vol 43: Mediterranean type ecosystems. Berlin.

LORA GONZÁLEZ, A., J.E. HERNÁNDEZ BERMEJO y J. PRADOS LIGERO -1998Cytisus malacitanus subsp. moleroi (Fern. Casas) A. Lora, Hern. Berm. y J. Prados (Fabaceae), stat. \& comb. nov.: posición taxonómica, corología y ecología. Act. Bot. Malacitana 23: 233-237.

RIVERA NÚÑEZ, D. y C. OBÓN DE CASTRO 1991- La guía de INCAFO de las plantas útiles y venenosas de la Península Ibérica y las Baleares (excluidas medicinales). Ed. INCAFO. Madrid.

U.I.C.N. -1981- Cómo usar las categorías del Libro Rojo de Datos de la U.I.C.N. Kew, Ritchmond.

U.I.C.N. -1994- Categorías de las Listas Rojas de la U.I.C.N. Comisión de Supervivencia de Especies de U.I.C.N. Kew, Ritchmond.

Aceptado para su publicación en Junio de 1998

Dirección de los autores. Jardín Botánico de Córdoba. Avda. de Linneo s/n. 14004 Córdoba. 